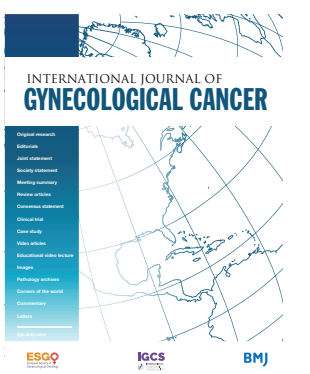

- Additional supplemental material is published online only. To view, please visit the journal online (http://dx.doi.org/ 10.1136/ijgc-2021-002983).

For numbered affiliations see end of article.

Correspondence to Dr Nobutaka Takahashi, Department of Gynecology, Shizuoka Cancer Center, Sunto-gun, Shizuoka, Japan; n. takahashi@scchr.jp

NT and KH contributed equally.

Received 3 August 2021 Accepted 13 0ctober 2021 Published Online First 1 November 2021

Check for updates

(C) IGCS and ESGO 2021. No commercial re-use. See rights and permissions. Published by BMJ.

To cite: Takahashi $\mathrm{N}$, Hatakeyama K, Nagashima T, et al. Int J Gynecol Cancer 2021;31:1557-1563.

\title{
Activation of oxidative phosphorylation in TP53-inactive endometrial carcinomas with a poor prognosis
}

Nobutaka Takahashi, ${ }^{1}$ Keiichi Hatakeyama, ${ }^{2}$ Takeshi Nagashima, ${ }^{3,4}$ Keiichi Ohshima, ${ }^{2}$ Kenichi Urakami, ${ }^{3}$ Ken Yamaguchi, ${ }^{5}$ Yasuyuki Hirashima ${ }^{1}$

\section{HIGHLIGHTS}

- TP53-inactive endometrial carcinomas with or without TP53 mutations have poor prognosis.

- Oxidative phosphorylation associating PI3K/mTOR and autophagy is activated in TP53-inactive tumors.

- Oxidative phosphorylation pathway is a potential drug target in endometrial carcinomas.

\section{ABSTRACT}

Objective We aimed to identify pathways for potential therapeutic targets by conducting molecular profiling of endometrial carcinomas in patients with poor prognosis. Methods The classification of endometrial carcinomas has undergone a paradigm shift with the advent of next generation sequencing based molecular profiling. Although this emerging classification reflects poor prognosis in patients with endometrial carcinoma, knowledge of affected biological pathways is still lacking. In this study, 85 patients with endometrial carcinomas at the Shizuoka Cancer Center were evaluated from January 2014 to March 2019 and classified based on The Cancer Genome Atlas subgroups. The accumulation of germline and somatic mutations was determined using next generation sequencing. Gene expression profiling was used to determine the effect of TP53 inactivation on the recurrence of endometrial carcinoma. Additionally, the biological pathways associated with TP53 inactivation were estimated by pathway analysis based on gene expression.

Results Based on The Cancer Genome Atlas classification, the ratio of polymerase-epsilon to copy number-high subgroups and the frequency of PTEN and TP53 mutations differed in patients, and mutations of ARHGAP35 observed in normal endometrium were accumulated in the polymerase-epsilon and microsatellite instability subgroups. We revealed that copy number-high reflects TP53 inactivation in endometrial carcinomas, and that TP53-inactive tumors with or without TP53 mutations have poor prognosis. Furthermore, overexpression of aurora kinase $A$ and activation of oxidative phosphorylation were found in TP53-inactivated endometrial carcinomas, suggesting that the PI3K/mTOR and autophagy pathways are potential drug targets.

Conclusion Our analysis revealed a relationship between pathways involved in oxidative phosphorylation and poor prognosis and provides insight into potential drug targets.

\section{INTRODUCTION}

WHO's Classification of Tumors of the Female Reproductive Organs, fourth edition, defines endometrial carcinoma, which accounts for the majority of uterine cancers, primarily by histological features and accompanying immunohistochemical markers. In the past decade, an emerging endometrial carcinoma classification has been proposed by The Cancer Genome Atlas $^{1}$ that classifies endometrial carcinomas into four subgroups based on molecular profiling using next generation sequencing: polymerase-epsilon, microsatellite instability, copy number-low, and copy number-high. However, molecular profiling of endometrial carcinoma has not been studied in Japanese patients.

Early-stage endometrial carcinoma is treated by hysterectomy with or without adjuvant therapy. Although advanced or recurrent endometrial carcinoma is mainly treated with cytotoxic anticancer drugs, the chemotherapeutic efficacy is limited and new treatments are needed. In a recent phase III KEYNOTE-775/Study 309 trial, the combination of lenvatinib and pembrolizumab significantly improved the overall survival of patients with endometrial carcinoma, regardless of tumor microsatellite instability status. ${ }^{2}$ However, the response rate of this treatment in copy number-high tumors, which have the poorest prognosis, remains unclear. Therefore, it is desirable to identify biological pathways and associated drug targets in the copy number-high subgroup to improve the prognosis of advanced or recurrent endometrial carcinomas.

According to previously reported molecular profiling, ${ }^{1}$ major characteristics of copy number-high tumors in endometrial carcinoma are TP53 mutation, amplification of MYC and ERBB2, and altered expression of cell cycle related genes and proteins. Multiple cohorts of inhibitors targeting the PI3K/AKT/mTOR pathway, which is closely associated with $M Y C$ aberration, have been tested in patients with endometrial carcinoma, but meta-analyses revealed no clear benefit in overall survival or tumor response rates. ${ }^{3}$ However, a randomized phase II trial with trastuzumab in conjunction with carboplatin and paclitaxel showed 


\section{Original research}

an improved progression-free and overall survival in patients with ERBB2-overexpressing uterine serous carcinoma, favoring the trastuzumab arm compared with chemotherapy alone. ${ }^{4}$ In the subgroup analysis of a phase II basket study (TAPUR trial), treatment with pertuzumab plus trastuzumab showed evidence of antitumor activity in heavily pretreated patients with uterine cancer and focal amplification of ERBB2 on chr17q12 or certain mutations. ${ }^{5}$ However, effective drugs for endometrial carcinomas without focal amplification of the $17 q 12$ region are not known. In this study, we conducted molecular profiling of endometrial carcinomas in Japanese patients with poor prognosis to identify pathways that could be potential therapeutic targets.

\section{METHODS}

Written informed consent was obtained from all patients, and the institutional review board at the Shizuoka Cancer Center approved all aspects of this study (authorization number 25-33). All experiments using clinical samples were performed in accordance with the approved Japanese ethical guidelines.

\section{Clinical Samples}

Endometrial carcinomas were extracted from the Japanese pancancer cohort (project HOPE-High-tech Omics-based Patient Evaluation) comprising 5521 tumor specimens (Online supplemental table S1). ${ }^{6}$ These samples were clinically diagnosed after surgery by a pathologist. Tumors and their surrounding tissues $(\geq 0.1 \mathrm{~g})$ were dissected from the surgical specimens immediately after resection of the lesion at the Shizuoka Cancer Center from January 2014 to March 2019. Additionally, peripheral blood was collected as a pair control to exclude germline mutations. The experimental protocols have been described previously. ${ }^{6}$

\section{Datasets for Analysis of Somatic Alteration}

We analyzed both sequencing and microarray data derived from freshly frozen clinical samples using our own previously constructed pipeline. ${ }^{6}$ In this study, a part of the dataset was extracted for analysis of somatic and germline alterations, including tumor mutational burden, copy number variations, mutational signatures, and gene expression signatures (Online supplemental table S2). Tiers 1 and 2 in somatic mutations were defined according to previously reported criteria. ${ }^{6}$ Mutational signature analysis was performed based on deconstructSigs, ${ }^{7}$ where deamination corresponds to single base substitutions 1 , polymerase-epsilon is the sum of single base substitutions $10 \mathrm{a}$ and $10 \mathrm{~b}$, and microsatellite instability is the sum of single base substitutions 6 , single base substitutions 14 , single base substitutions 15 , single base substitutions 20 , single base substitutions 21 , single base substitutions 26 , and single base substitutions 44 in the Catalog of Somatic Mutations in Cancer mutational signatures (v3, https://cancer.sanger.ac.uk/cosmic/ signatures).

The contribution of mutational signatures was calculated only for tumors, excluding those with $<50$ mutations. Somatic copy number variations were detected using SAAS copy number variations. ${ }^{8}$ This method accounted for the read-depth ratio and B allele frequency, and achieved the best performance among the six copy number variation detection tools. ${ }^{9}$ The copy number variation size was calculated from the sum of the loss (copy number variations $\leq 1.5$ ), gain (copy number variations $\geq 2.5$ ), and copy neutral loss of heterozygosity in the genome, estimated by whole exome sequencing. To compare our platform with others, mutation and expression profiles of endometrial carcinomas were extracted from the public database in The Cancer Genome Atlas project (Pan-Cancer Atlas). ${ }^{10}$

\section{Gene Expression Analysis}

For RNA analysis, tumor tissues were immediately immersed in RNAlater (Thermo Fisher Scientific). Purified total RNA was amplified and fluorescent-labeled using a One-Color Low Input Quick Amp Labeling Kit (Agilent Technologies, Santa Clara, CA). Cy3labeled cRNAs were hybridized to a SurePrint G3 Human Gene Expression $8 \times 60 \mathrm{~K}$ v2 Microarray (Agilent Technologies), containing 50599 probes, representing 29833 genes registered in the Entrez Gene Database. Expression signature was calculated from the average of genes in unique gene sets corresponding to each individual signature (TP53 inactivation, ${ }^{11} \mathrm{~T}$ cell inflamed gene expression profile, ${ }^{12}$ epithelial-mesenchymal transition, ${ }^{13} \mathrm{PI} 3 \mathrm{~K} / \mathrm{mTOR},{ }^{14}$ and autophagy ${ }^{15}$ ). Pathway analysis was performed based on gene set enrichment analysis ${ }^{16}$ using the Hallmark gene set (version 7.2).

\section{Statistical Analysis}

Fisher's exact test was performed to detect mutations and clinical characteristics, except for age. The exact test for the mutations was performed separately for the two groups consisting of high tumor mutational burden and the remaining two groups because of large bias in the tumor mutational burden of the four groups. For comparison of age and expression signatures, one-way analysis of variance and Welch's t test were performed, respectively. The log-rank test was performed for relapse-free survival curves. The statistical analysis was calculated and visualized using $R$ (version 4.0.2). To control the false discovery rate in gene set enrichment analysis, the Benjamini-Hochberg procedure $(q<0.05)$ was performed, and results were considered significant at $p<0.01$.

\section{RESULTS}

\section{Molecular Classification}

Eighty-five endometrial carcinomas with detailed clinical information were extracted from the Japanese version of The Cancer Genome Atlas data cohort, consisting of 5521 tumor specimens. ${ }^{7}$ Among these, $82 \%$ (70/85) were endometrioid carcinomas and $61 \%$ $(43 / 70)$ of the endometrioid carcinomas were grade 1 (Figure 1A). These tumors were divided into four subgroups according to The Cancer Genome Atlas classification ${ }^{1}$ (Figure 1B); copy number-high was defined based on copy number variations in all chromosomes (Online supplemental figure S1). No significant differences in clinicopathological factors were observed between the subgroups (Online supplemental table S1). The endometrial carcinomas in Japanese patients tended to have more polymerase-epsilon subgroups and fewer copy number-highs than The Cancer Genome Atlas classification, and clear cell carcinomas were present in all subgroups except copy number-high.

\section{Molecular Profiling}

The molecular profiling of the subgroups, classified according to The Cancer Genome Atlas, was investigated in 85 endometrial carcinomas. Germline and somatic mutations and mutational 
(A)

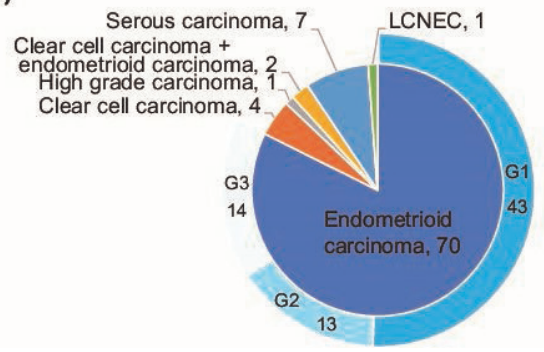

(B)

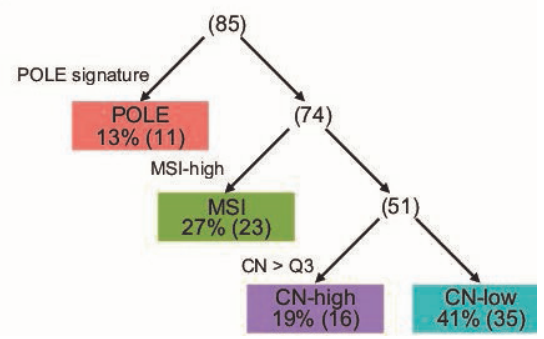

Figure 1 Sample profile of endometrial carcinomas. (A) Distribution of tumor type in 85 samples. LCNEC, large cell neuroendocrine carcinoma. (B) Classification of endometrial carcinomas based on The Cancer Genome Atlas profiling of endometrial carcinomas. CN, copy number; MSI, microsatellite instability; POLE, polymerase-epsilon; Q3, 75th percentile.

signatures were displayed based on whole exome sequencing and target panel sequencing (Figure 2 and Online supplemental table S2). Consistent with a previous report, ${ }^{1}$ the polymerase-epsilon and microsatellite instability subgroups had higher tumor mutational burden, and endometrial carcinomas in the copy number-low subgroup harbored significant accumulation of somatic mutations in PTEN and ARID1A, whereas TP53 mutations were accumulated in the copy number-high subgroup. Accumulation of ARHGAP35 mutations observed in the normal endometrial epithelium was more common in the polymerase-epsilon and microsatellite instability subgroups. No specific mutational signature was detected, except for the polymerase-epsilon and microsatellite instability signatures.

These subgroups were clinically characterized. The copy numberhigh subgroup had a worse prognosis than the other subgroups (Online supplemental figure S2A). The T cell inflamed signature, which predicts the effect of immune checkpoint inhibition, ${ }^{17}$ was significantly lower in copy number-high than in polymerase-epsilon and microsatellite instability subgroups, and tended to be lower in copy number-high than in copy number-low subgroups (Online supplemental figure S2B). Copy number-low accounted for more than $50 \%$ of grade 1 endometrioid carcinomas, while approximately $57 \%$ of serous carcinomas were classified as copy numberhigh (Online supplemental figure S2C).

\section{Classification Using TP53 Inactivation}

We analyzed the inactivation and somatic mutations of TP53 in endometrial carcinomas based on gene expression profiling and whole exome sequencing. Relapses were more frequent in tumors with a high TP53 inactivation score or TP53 mutation accumulation (Figure 3). The copy number-high and copy number-low subgroups in The Cancer Genome Atlas classification were altered to TP53active and TP53-inactive subgroups based on TP53 inactivation score and mutations, resulting in $63 \%$ of copy number-high being classified as TP53-inactive and $89 \%$ of copy number-low being classified as TP53-active subgroup (Figure 4A). Furthermore, this TP53-inactive subgroup had an extremely poor prognosis (median survival time 20 months; Figure 4B). No significant difference in the $T$ cell inflamed signature was observed between the TP53active and TP53-inactive subgroups (Online supplemental figure $\mathrm{S} 3 \mathrm{~A})$. More than $65 \%$ of grade 1 endometrioid carcinomas and

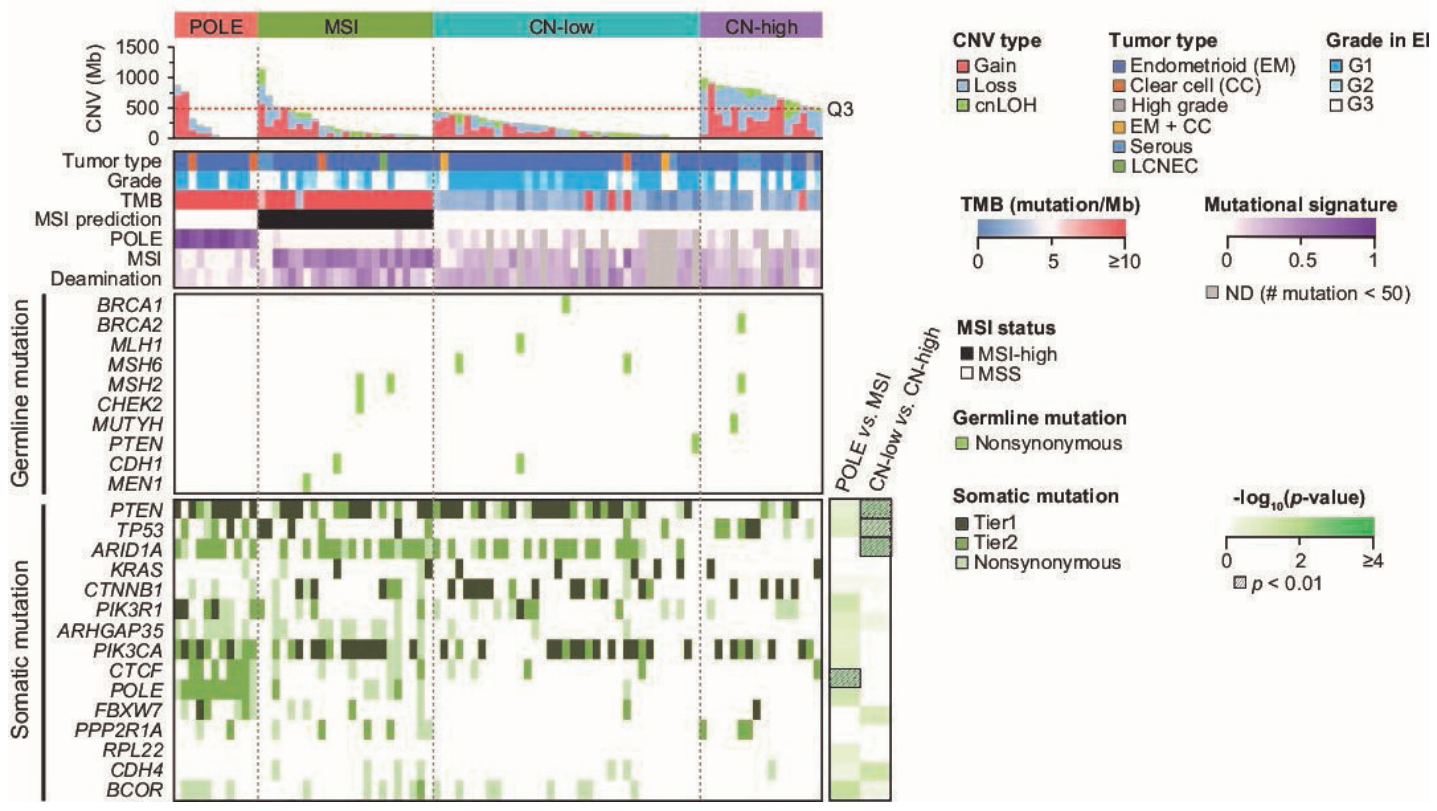

Figure 2 Molecular profiling of endometrial carcinomas. The samples are classified based on The Cancer Genome Atlas profiling, and they are sorted in descending order according to copy number variation (CNV) size. The 75th percentile (Q3) of CNV size is represented by the red dotted line. 


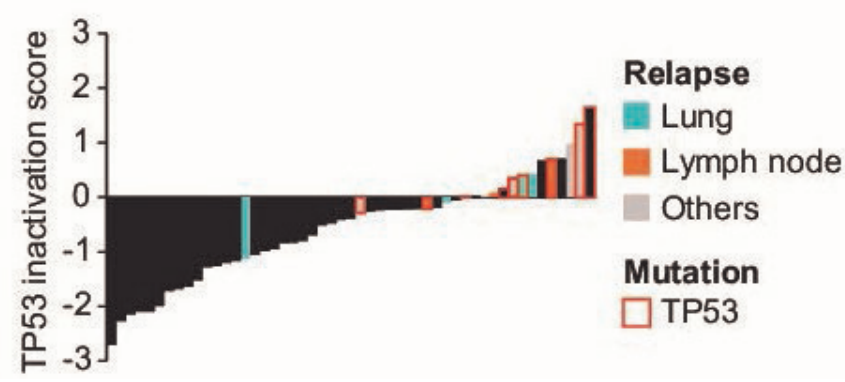

Figure 3 Relationship between TP53 inactivation score and relapse in endometrial carcinomas excluding polymeraseepsilon and microsatellite instability. The samples are sorted in ascending order of TP53 inactivation score. Relapses are colored other than black, and samples with TP53 nonsynonymous mutations are represented by red boxes.

approximately $71 \%$ of serous carcinomas were classified as TP53 active and TP53 inactive, respectively (Online supplemental figure S3B).

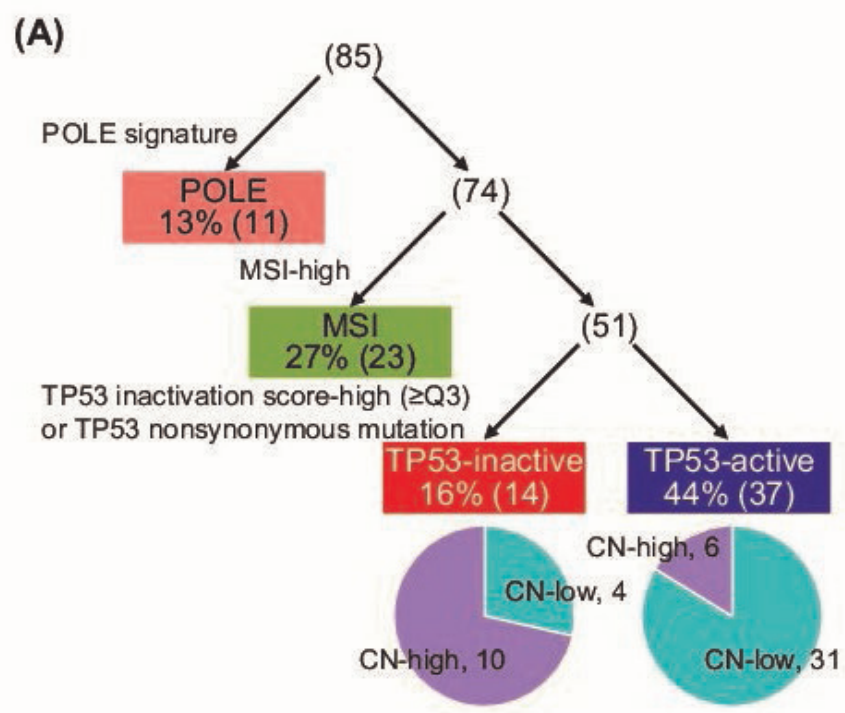

(B)

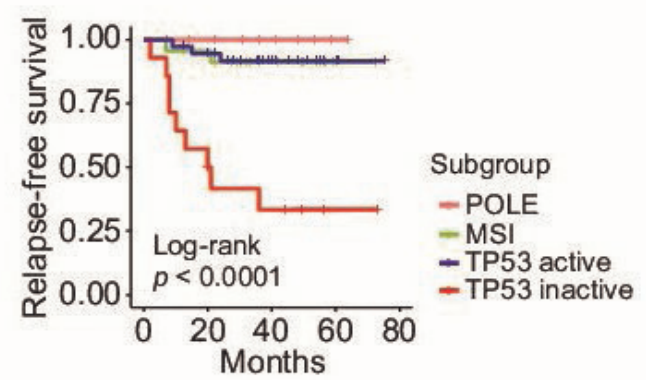

Figure 4 Characteristics of TP53-inactivated endometrial carcinomas based on gene expression profiling. (A) New classification of endometrial carcinomas. Pie charts represent the distribution of The Cancer Genome Atlas classification within the TP53 inactive and active subgroups. (B) Relapse-free survival curve of patients in new subgroups, including TP53 status. MSI, microsatellite instability; POLE, polymerase-epsilon.

\section{Gene Expression Between TP53-active and TP53-inactive Carcinomas}

Gene set enrichment analysis of the TP53-active and TP53-inactive subgroups predicted the biological pathways affected by TP53 inactivation in endometrial carcinomas. Among the significantly enriched pathways in "Hallmark" gene sets, 90\% (9/10) were reported in the TP53-inactivated population in 11 cancer types $^{11}$ (Figure 5A). Moreover, oxidative phosphorylation was significantly enriched in endometrial carcinoma. The expression of 11 core genes that were upregulated in $>1 / 3$ of the 10 pathways found in the gene set enrichment analysis is shown along with the copy number variations of the cytoband in which the genes are located (Figure 5B). Gene expression of AURKA, BUB1, CCNB2, CDK1, KIF2C, MCM2, and MCM4 was strongly correlated with the TP53 inactivation score $(r 0.8)$. Copy number amplification of cytobands encoding BUB1 (2q13), MYC (8q24.21), and RFC4 (3q27.3) was observed in tumors with high TP53 inactivation scores, whereas no copy number alterations were detected in other genes. These results suggest that gene expression of AURKA, CCNB2, CDK1, KIF2C, MCM2, and MCM4, but not BUB1, is associated with TP53 inactivation independent of copy number gain in Japanese patients with endometrial carcinoma.

To clarify the biological pathways that activate oxidative phosphorylation in TP53-inactivated tumors, we examined the correlation between TP53 inactivation and known oxidative phosphorylation related expression signatures (epithelial-mesenchymal transition, $\mathrm{PI} 3 \mathrm{~K} / \mathrm{mTOR}$ and autophagy). A significant correlation between TP53 inactivation and the oxidative phosphorylation-related signatures, excluding epithelial-mesenchymal transition, was observed (Figure 5C and Online supplemental table S2).

\section{Validation of TP53-active and TP53-inactive Subgroups}

We reclassified endometrial carcinomas using The Cancer Genome Atlas dataset. The TP53-inactive subgroup accounted for $31.2 \%$ of all tumors, which was more than that observed in our cohort, reflecting the frequency of copy number-high in the Cancer Genome Atlas dataset (Online supplemental figure S4A). In The Cancer Genome Atlas dataset, the TP53-inactive subgroup had poor prognosis compared with the other subgroups (Online supplemental figure S4B). Furthermore, the expression patterns of the core genes identified from the gene set enrichment analysis were strongly correlated with the TP53 inactivation score (Online supplemental figure S4C).

\section{DISCUSSION}

\section{Summary of Main Results}

Based on The Cancer Genome Atlas classification, the ratio of polymerase-epsilon to copy number-high subgroups was different in Japanese patients, and ARHGAP35 mutations observed in normal endometrium were accumulated in the polymerase-epsilon and microsatellite instability subgroups. copy number-high reflected TP53 inactivation in endometrial carcinomas, and the TP53-inactive subgroup had extremely poor prognosis. Furthermore, oxidative phosphorylation was activated in TP53-inactive endometrial carcinomas, and $A U R K A$, a potential drug target, was highly expressed regardless of copy number gain. The mutation profile and prognosis of Japanese patients were consistent with those in The Cancer 
(A)

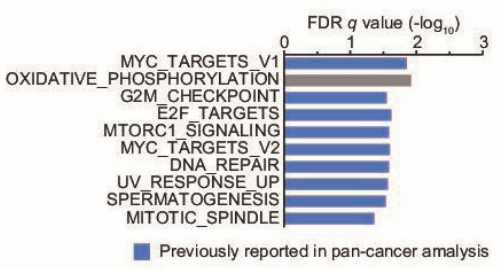

(C)

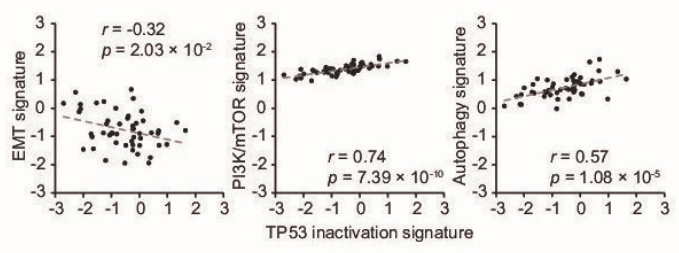

(B)

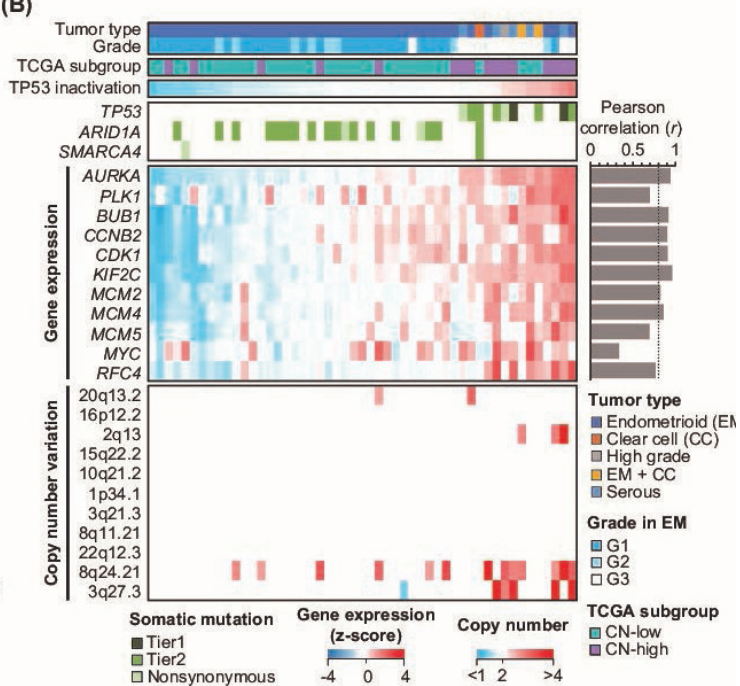

Figure 5 Characteristics of the TP53-inactive subgroup based on gene expression profiling. (A) Gene set enrichment analysis (GSEA) between TP53-active and TP53-inactive tumors. (B) gene expression and copy number variation (CNV) of core genes in the gene set of GSEA. The CNVs were selected from the cytobands where those genes were located. (C) correlation with TP53 inactivation signature. epithelial-mesenchymal transition (EMT), PI3K/mTOR, and autophagy signatures were calculated using the corresponding gene sets.

Genome Atlas ${ }^{1}$ suggesting that The Cancer Genome Atlas classification could be reproduced in Japanese patients with endometrial carcinoma using our next generation sequencing pipeline.

\section{Results in the Context of Published Literature}

Asians tend to harbor fewer TP53 mutations and more PTEN mutations according to a reanalysis based on The Cancer Genome Atlas data. ${ }^{18}$ Somatic mutations in PTEN are often observed in colorectal cancers and endometrial carcinomas with dysfunctional polymerase-epsilon, ${ }^{1}$ and TP53 mutations in endometrial carcinoma are common in the copy number-high subgroup. ${ }^{1}$ Therefore, the difference in the proportions of polymerase-epsilon and copy number-high subgroups may be a characteristic of Japanese or Asian populations.

In endometrial carcinomas, germline and somatic mutations were investigated based on blood and tumor whole exome sequencing. No subgroup harbored specific germline mutations, whereas somatic mutations in ARHGAP35 accumulated in the polymeraseepsilon and microsatellite instability subgroups. ARHGAP35 was reported to be altered in endometriosis and normal endometrium. ${ }^{16}$ Although ARHGAP35 is associated with the promoter region of the glucocorticoid receptor gene, ${ }^{19}$ its relationship with defective polymerase-epsilon and chromosomal instability, including microsatellite instability, has not been reported. Hence, ARHGAP35 mutations in endometrial carcinoma may accumulate in normal cells, even before cancer development.

A combination of tumor mutational burden and T-cell activation related gene expression creates a signature that can improve the prediction of responses to immune checkpoint inhibitors. ${ }^{12}$ We found that this signature was higher in the polymerase-epsilon and microsatellite instability subgroups with high tumor mutational burden, suggesting that immune checkpoint inhibitors may be effective against grade 3 endometrioid carcinomas, of which these subgroups account for $70 \%$. Therefore, the application of a combination of immune checkpoint inhibitors and antiangiogenics for endometrial carcinoma may be effective in the treatment of grade 3 endometrioid carcinomas.

We found predominantly TP53-inactive tumors in the copy number-high subgroup, and the TP53-inactive subgroup showed an extremely poor prognosis. Although clear cell carcinomas were not included in the copy number-high subgroup, TP53 inactivation progressed in these tumors (Figures 2 and 4B). TP53 mutation is related to malignancy in endometrial carcinomas without polymerase-epsilon mutations or mismatch repair deficiencies. ${ }^{20}$ As TP53 dysfunction is one of the triggers for chromosomal aberrations in various cancers, ${ }^{1121}$ it is reasonable to assume that TP53 pathway inactivation is involved in endometrial carcinoma malignancy before chromosomal instability. Additionally, the prognosis of the TP53-active subgroup was similar to that of the microsatellite instability subgroup, with few recurrences, whereas the TP53-inactive subgroup had poor prognosis, with many grade 3, serous, and clear cell carcinomas. In The Cancer Genome Atlas cohort, grade 3 endometrioid carcinomas also accumulated TP53 mutations, ${ }^{1}$ and in another Japanese cohort, grade 3 with TP53 aberrations showed a poor prognosis. ${ }^{22}$ Therefore, drug discovery focusing on TP53-inactive populations with or without TP53 mutations will provide a new perspective for the treatment of endometrial carcinoma.

Patients with TP53-inactivated tumors with upregulated oxidative phosphorylation had an extremely poor prognosis and a reduced T-cell inflamed gene expression profile signature (including CD8 expression) in the tumor microenvironment. Oxidative phosphorylation activation in a tumor invokes metabolic insufficiency by nutrient competition and intercellular exchanges in the tumor microenvironment, leading to $\mathrm{CD} 8+\mathrm{T}$ cell dysfunction. ${ }^{23}$ Patients with oxidative phosphorylation and CD8+ squamous cell carcinoma of the lung and craniocervical region have a poor prognosis. ${ }^{24}$ Tumor microenvironment and oxidative phosphorylation may have an important role in endometrial carcinoma tumor progression. 


\section{Original research}

Pathway analysis revealed that oxidative phosphorylation related genes are enriched in the TP53-inactive subgroup of endometrial carcinomas, which was not found in a previous report on TP53 inactivation in multiple cancer types. ${ }^{11}$ The pathway involved in oxidative phosphorylation is underutilized in many cancer cells because of alterations in intracellular metabolism, as exemplified by the Warburg effect. ${ }^{25}$ Our analysis found that the expression of several core genes, including AURKA, correlated strongly with the TP53 inactivation score, independent of copy number. AURKA is incorporated into the mitochondrial matrix during interphase, influencing mitochondrial morphology, ${ }^{26}$ and its overexpression promotes metabolic reprogramming by increasing the mitochondrial interconnectivity involved in oxidative phosphorylation progression. ${ }^{27}$ Taken together, AURKA is highly upregulated in endometrial carcinomas with TP53-inactive cells, resulting in oxidative phosphorylation activation.

In lung cancer, SWI/SNF deficiencies caused by $A R I D 1 A$ and SMARCA4 mutations induce oxidative phosphorylation. ${ }^{28}$ However, accumulation of somatic mutations in these genes was not found in the TP53-mutated endometrial carcinomas (Figure 5B). Epithelialmesenchymal transition, which is negatively correlated with oxidative phosphorylation based on profiling of several cancers ${ }^{29}$ was not significantly correlated with the TP53 inactivation signature. Interestingly, the signatures of the activated PI3K/mTOR and autophagy pathways that can induce oxidative phosphorylation ${ }^{30}$ were positively correlated with that of TP53 inactivation. Based on wholegenome sequencing analysis of 2658 cancers, TP53 inactivation is estimated to occur early in uterine cancer development, followed by amplification in the MYC-containing chromosome $8 \mathrm{q}$ and other genomic alterations. ${ }^{31}$ Therefore, oxidative phosphorylation may be proceeded by $\mathrm{PI} 3 \mathrm{~K} / \mathrm{mTOR}$ and autophagy activation triggered by TP53 inactivation in endometrial carcinoma.

\section{Strengths and Weaknesses}

Our classification allowed us to extract a population with a worse prognosis than chromosomal instability and to propose a new drug candidate for oxidative phosphorylation in endometrial carcinoma. This molecular profiling approach can provide a bird's eye view of the genomic features in the tumor, but needs to be further validated using TP53-inactivated cell lines and mouse models to evaluate the efficacy of candidate drugs.

\section{Implications for Practice and Future Research}

In TP53-inactivated endometrial carcinoma, oxidative phosphorylation was not only activated by over-expression of $A U R K A$, but also by the PI3K/mTOR and autophagy pathways, suggesting its important role in this tumor. Recently, the combination of paclitaxel and alisertib, an AURKA inhibitor, prolonged progression-free survival in ovarian cancer. ${ }^{32}$ The efficacy of alisertib in human papillomaviruspositive cervical cancer has also been reported. ${ }^{33}$ Additionally, IACS-010759, a clinical-grade small molecule targeting mitochondrial respiratory chain complex I, was recently reported as an oxidative phosphorylation inhibitor. ${ }^{28}$ Therefore, a combination of AURKA and oxidative phosphorylation inhibitors may be effective in TP53-inactivated endometrial carcinomas with high AURKA expression and activated oxidative phosphorylation by the PI3K/mTOR and autophagy pathways. Further experiments using a xenograft model of TP53-inactivated cell lines are needed in future studies.
Recently, WEE1-like protein kinase inhibitors have also attracted attention as promising drugs for serous endometrial carcinomas. ${ }^{34}$ Inhibition of WEE1 with adavocertib disrupts the Gap2/Mitotic phase arrest required for DNA damage repair and increases replication stress. ${ }^{35}$ In cells with altered Gap1/Synthesis checkpoints with occurring p53 dysfunction, adavocertib induces premature mitosis and subsequent apoptosis due to unrepaired DNA damage because of the high reliance on the Gap2/Mitotic checkpoint. Therefore, an accurate understanding of the inactivation state of TP53 in endometrial carcinomas is important in determining the therapeutic strategy for this new agent as well as for AURKA and oxidative phosphorylation inhibitors.

\section{CONCLUSION}

Our analysis showed that the The Cancer Genome Atlas classification is reproducible in Japanese patients with endometrial carcinoma, revealed a relationship between pathways involved in oxidative phosphorylation and poor prognosis, and provided insights into potential drug targets.

\section{Author affiliations}

${ }^{1}$ Department of Gynecology, Shizuoka Cancer Center, Sunto-gun, Shizuoka, Japan ${ }^{2}$ Medical Genetics Division, Shizuoka Cancer Center Research Institute, Sunto-gun, Shizuoka, Japan

${ }^{3}$ Cancer Diagnostics Research Division, Shizuoka Cancer Center Research Institute, Sunto-gun, Shizuoka, Japan

${ }^{4}$ SRL Inc, Shinjuku-ku, Tokyo, Japan

${ }^{5}$ Shizuoka Cancer Center, Sunto-gun, Shizuoka, Japan

Acknowledgements We thank the members of the Shizuoka Cancer Center Hospital and Research Institute for their support and suggestions. This work was supported by the Shizuoka Prefectural Government of Japan. We would like to thank Editage (www.editage.jp) for English language editing.

Contributors NT is responsible for the overall content as the guarantor. NT and KH contributed equally to this work and designed the study. KH, TN, KO, KU performed the experiments. $\mathrm{KH}$ and NT analyzed the data. TN and KH performed statistical analyses. $\mathrm{KH}$ and NT wrote the paper, with contributions from KY and YH. All authors have read and approved the final version of the manuscript.

Funding The authors have not declared a specific grant for this research from any funding agency in the public, commercial or not-for-profit sectors.

Competing interests None declared.

Patient consent for publication Consent obtained directly from patient(s)

Ethics approval The institutional review board of the Shizuoka Cancer Center, authorization number: 25-33.

Provenance and peer review Not commissioned; externally peer reviewed.

Data availability statement Data are available upon reasonable request. Data are available in a public, open access repository. The authors declare that all data supporting the findings of this study are available within the article, its supplementary information files, and from the corresponding author upon reasonable request. In accordance with the journal's guidelines, the data were submitted to the National Bioscience Database Center Human Database as "Controlled-Access Data" (Research ID, hum0127, https://humandbs. biosciencedbc.jp/en/).

Supplemental material This content has been supplied by the author(s). It has not been vetted by BMJ Publishing Group Limited (BMJ) and may not have been peer-reviewed. Any opinions or recommendations discussed are solely those of the author(s) and are not endorsed by BMJ. BMJ disclaims all liability and responsibility arising from any reliance placed on the content. Where the content includes any translated material, BMJ does not warrant the accuracy and reliability of the translations (including but not limited to local regulations, clinical guidelines, terminology, drug names and drug dosages), and is not responsible for any error and/or omissions arising from translation and adaptation or otherwise. 


\section{REFERENCES}

1 Cancer Genome Atlas Research Network, Kandoth C, Schultz N, et al. Integrated genomic characterization of endometrial carcinoma. Nature 2013;497:67-73.

2 Makker V, Taylor $\mathrm{MH}$, Aghajanian C, et al. Lenvatinib plus pembrolizumab in patients with advanced endometrial cancer. J Clin Oncol 2020;38:2981-92.

3 Roncolato F, Lindemann K, Willson ML, et al. Pi3K/Akt/mTOR inhibitors for advanced or recurrent endometrial cancer. Cochrane Database Syst Rev 2019;10.

4 Fader AN, Roque DM, Siegel E, et al. Randomized phase II trial of carboplatin-paclitaxel compared with Carboplatin-PaclitaxelTrastuzumab in advanced (stage III-IV) or recurrent uterine serous carcinomas that overexpress HER2/neu (NCT01367002): updated overall survival analysis. Clin Cancer Res 2020;26:3928-35.

5 Ali-Ahmad HM, Rothe M, Mangat PK, et al. Pertuzumab plus trastuzumab $(\mathrm{P}+\mathrm{T})$ in patients (Pts) with uterine cancer (UC) with $E R B B 2$ or ERBB3 amplification, overexpression or mutation: Results from the Targeted Agent and Profiling Utilization Registry (TAPUR) study. Journal of Clinical Oncology 2021;39:5508.

6 Nagashima T, Yamaguchi K, Urakami K, et al. Japanese version of the cancer genome atlas, JCGA, established using fresh frozen tumors obtained from 5143 cancer patients. Cancer Sci 2020;111:687-99.

7 Rosenthal R, McGranahan N, Herrero J, et al. DeconstructSigs: delineating mutational processes in single tumors distinguishes DNA repair deficiencies and patterns of carcinoma evolution. Genome Biol 2016;17:31.

8 Zhang Z, Hao K. SAAS-CNV: a joint segmentation approach on aggregated and allele specific signals for the identification of somatic copy number alterations with next-generation sequencing data. PLoS Comput Biol 2015;11:e1004618.

$9 \mathrm{Kim} \mathrm{H}-\mathrm{Y}$, Choi J-W, Lee J-Y, et al. Gene-based comparative analysis of tools for estimating copy number alterations using whole-exome sequencing data. Oncotarget 2017;8:27277-85.

10 Hoadley KA, Yau C, Hinoue T, et al. Cell-of-origin patterns dominate the molecular classification of 10,000 tumors from 33 types of cancer. Cell 2018;173:291-304.

11 Donehower LA, Soussi T, Korkut A, et al. Integrated analysis of TP53 gene and pathway alterations in the cancer genome atlas. Cell Rep 2019;28:1370-84.

12 Ayers M, Lunceford J, Nebozhyn M, et al. IFN- $\gamma$-related mRNA profile predicts clinical response to PD-1 blockade. J Clin Invest 2017;127:2930-40.

13 Cristescu R, Lee J, Nebozhyn M, et al. Molecular analysis of gastric cancer identifies subtypes associated with distinct clinical outcomes. Nat Med 2015;21:449-56.

14 Zhang Y, Kwok-Shing Ng P, Kucherlapati M, et al. A pan-cancer Proteogenomic atlas of PI3K/Akt/mTOR pathway alterations. Cancer Cell 2017;31:820-32.

15 Huang Z, Liu J, Luo L, et al. Genome-Wide identification of a nove autophagy-related signature for colorectal cancer. Dose Response 2019;17:1559325819894179.

16 Suda K, Nakaoka H, Yoshihara K, et al. Clonal expansion and diversification of cancer-associated mutations in endometriosis and normal endometrium. Cell Rep 2018;24:1777-89.

17 Ott PA, Bang Y-J, Piha-Paul SA, et al. T-cell-inflamed geneexpression profile, programmed death ligand 1 expression, and tumor mutational burden predict efficacy in patients treated with pembrolizumab across 20 cancers: KEYNOTE-028. J Clin Oncol 2019;37:318-27.

18 Guttery DS, Blighe K, Polymeros K, et al. Racial differences in endometrial cancer molecular portraits in the cancer genome atlas. Oncotarget 2018;9:17093-103.

19 Lévay M, Settleman J, Ligeti E. Regulation of the substrate preference of p190RhoGAP by protein kinase C-mediated phosphorylation of a phospholipid binding site. Biochemistry 2009;48:8615-23.

20 Stelloo E, Nout RA, Osse EM, et al. Improved risk assessment by integrating molecular and clinicopathological factors in early-stage endometrial Cancer-Combined analysis of the PORTEC cohorts. Clin Cancer Res 2016;22:4215-24.

21 Smith ML, Fornace AJ. Genomic instability and the role of p53 mutations in cancer cells. Curr Opin Oncol 1995;7:68-75.

22 Yano M, Ito K, Yabuno A, et al. Impact of TP53 immunohistochemistry on the histological grading system for endometrial endometrioid carcinoma. Mod Pathol 2019;32:1023-31.

23 Reina-Campos M, Scharping NE, Goldrath AW. CD8 ${ }^{+}$T cell metabolism in infection and cancer. Nat Rev Immunol 2021. doi:10.1038/s41577-021-00537-8. [Epub ahead of print: 12 May 2021].

24 Frederick M, Skinner HD, Kazi SA, et al. High expression of oxidative phosphorylation genes predicts improved survival in squamous cell carcinomas of the head and neck and lung. Sci Rep 2020;10:6380.

25 Koppenol WH, Bounds PL, Dang CV. Otto Warburg's contributions to current concepts of cancer metabolism. Nat Rev Cancer 2011:11:325-37.

26 Grant R, Abdelbaki A, Bertoldi A, et al. Constitutive regulation of mitochondrial morphology by Aurora a kinase depends on a predicted cryptic targeting sequence at the $\mathrm{N}$-terminus. Open Biol 2018;8:170272.

27 Bertolin G, Bulteau A-L, Alves-Guerra M-C, et al. Aurora kinase A localises to mitochondria to control organelle dynamics and energy production. Elife 2018;7:e38111.

28 Lissanu Deribe Y, Sun Y, Terranova C, et al. Mutations in the SWI/ SNF complex induce a targetable dependence on oxidative phosphorylation in lung cancer. Nat Med 2018;24:1047-57.

29 Gaude E, Frezza C. Tissue-specific and convergent metabolic transformation of cancer correlates with metastatic potential and patient survival. Nat Commun 2016;7:13041.

30 Bosc C, Broin N, Fanjul M, et al. Autophagy regulates fatty acid availability for oxidative phosphorylation through mitochondriaendoplasmic reticulum contact sites. Nat Commun 2020;11:4056.

31 Gerstung M, Jolly C, Leshchiner I, et al. The evolutionary history of 2,658 cancers. Nature 2020;578:122-8.

32 Falchook G, Coleman RL, Roszak A, et al. Alisertib in combination with weekly paclitaxel in patients with advanced breast cancer or recurrent ovarian cancer: a randomized clinical trial. JAMA Oncol 2019;5:e183773.

33 Gabrielli B, Bokhari F, Ranall MV, et al. Aurora A is critical for survival in HPV-Transformed cervical cancer. Mol Cancer Ther 2015;14:2753-61.

34 Liu JF, Xiong N, Campos SM, et al. Phase II study of the WEE1 inhibitor adavosertib in recurrent uterine serous carcinoma. J Clin Oncol 2021;39:1531-9.

35 Fu S, Wang Y, Keyomarsi K, et al. Strategic development of AZD1775, a Wee1 kinase inhibitor, for cancer therapy. Expert Opin Investig Drugs 2018;27:741-51. 